\title{
DERMATITIS HERPETIFORMIS AND THYROTOXICOSIS
}

\author{
JEFFREY P. CALLEN, M.D., WILLIAM F. WESTON, M.D. AND JOSEPH J. CHANDA, M.D.
}

\begin{abstract}
Dermatitis herpetiformis has been associated with a variety of thyroid abnormalties. A case of thyrotoxicosis in a patient with pre-existing dermatitis herpetiformis is reported. Thyroid antibodies were present in the serum. This may suggest an immunologic relationship between dermatitis herpetiformis and thyroid disorders, that may be more than fortuitous.
\end{abstract}

Thyroid diseases have been reported to be associated with dermatitis herpetiformis $(\mathrm{DH})$ 1 $^{1-8}$ Little is known about the pathogenesis of this association. It is possible that iodine metabolism is abnormal in $\mathrm{DH}$ or may predispose to the development of $\mathrm{DH}$ in a susceptible individual. 5 On the other hand, an autoimmune event may be the process which could link these two conditions, because each of these conditions is well recognized to be associated with other autoimmune phenomena. We report a case of Graves disease associated with thyroid antibodies in a patient with a long history of dermatitis herpetiformis. We believe that the association of these conditions has been more frequently reported than coincidence would allow.

Address for reprints: Jeffrey P. Callen, M.D., 323 East Chestnut Street, Louisville, KY 40202.
From the Departments of Dermatology,

University of Louisville School of Medicine, Louisville, Kentucky, and the University of Michigan, Ann Arbor, Michigan

\section{Case Report}

CR was first seen at the University of Michigan Medical Center in 1969 at age 13 for evaluation of persistent pruritus. Initial history was noncontributory. Physical examination revealed symmetrical excoriations without primary lesions (Fig. 1). No grouping was found. The opinion was that he had "neurodermatitis" and he was treated with topical corticosteroids. The patient was unresponsive and on return appointment he was noted to have small grouped vesicular lesions on symmetrical extensor surfaces. A skin biopsy specimen of a vesicle showed a subepidermal separation with papillary infiltration with both polymorphonuclear leukocytes and eosinophils (Fig. 2). The biopsy was felt to be typical for dermatitis herpetiformis. Neither immunofluorescent studies, nor studies of gastrointestinal function were done. A G6PD level was normal. The patient was begun on diaminodiphenyl sulfone (DDS-dapsone) $100 \mathrm{mg}$ twice per day with complete resolution of the rash and symptoms. The patient was placed on a maintenance dose of DDS between 100-150 mg/day and remained well until 1975 .

In March 1975, the patient noted increased sweating, weight loss of 15 pounds, fatigue, nervousness, mild diarrhea and palpitations. He felt that these symptoms were due to the dapsone and discontinued the dapsone. The skin rash and pruritus returned and the patient was again evaluated in the dermatology clinic in July 1975. 


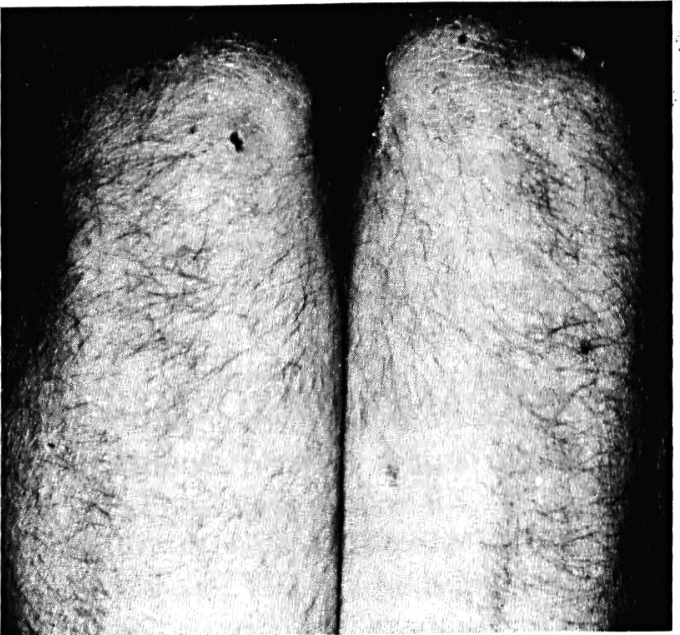

Fig. 1. Symmetrical grouped excoriations.

On physical examination the resting pulse was 124 beats per minute, blood pressure $14 / 180$, temperature $98.4 \mathrm{~F}$. A fine tremor, moderate palmar sweating, and a slight lid lag was noted. Exopthalmus was not present. Examination of the skin showed occasional scattered vesicular grouped lesions and multiple excoriated areas. A marked enlargement of the thyroid gland was noted. No bruit was heard over the thyroid: there was no lymphadenopathy. The remainder of the physical examination was normal.

Laboratory evaluation disclosed the following abnormalities: $T_{3}$ resin exchange $=149 \%$ (normal $=$ $86-114 \% ; T_{4}$ by Murphy-Patee $=16.6$ (normal-4.4-
$9.8 \mu \mathrm{g} / \mathrm{dl}$ ). Radioactive iodine uptake was $57.8 \%$ (normal-7-30\%). Thyroid antibodies were positive (1.25 titer). The remainder of the laboratory evaluation included $\mathrm{CBC}$, multiple screening test, urinalysis, chest $x$-ray examination was normal.

The patient was placed on methimazole $20 \mathrm{mg}$ three times per day, propranolol $40 \mathrm{mg}$ four times per day and dapsone $100 \mathrm{mg}$ per day. The rash and pruritus were controlled. However, the symptoms of hyperthyroidism persisted. In December 1975, a subtotal thyroidectomy was performed. Histologic study revealed benign thyroid hyperplasia. Immunofluorescent studies were not performed. In the year following the thyroidectomy, the patient has been euthyroid requiring no medications. His $\mathrm{DH}$ has been controlled with only $50 \mathrm{mg}$ of dapsone every other day.

\section{Comment}

Our patient has both dermatitis herpetiformis and hyperthyroidism. The diagnosis of $\mathrm{DH}$ was made based on typical clinical history, physical examination, histology and response to dapsone. ${ }^{9}$ Immunofluorescent studies were not available at the time of initial diagnosis and were not deemed necessary for the patient's care in 1975, and were therefore not accomplished. The diagnosis of hyperthyroidism was established by the marked elevation in thyroid function tests and typical histologic examination.

The association of $\mathrm{DH}$ and thyroid disor-



Fig. 2. Papillary microabscess with subepidermal separation, typical of dermatitis herpetiformis (H.\&E. $\times$ 100). 
ders has recently been reviewed by Douglas and Alexander. ${ }^{5}$ They reported four cases of $\mathrm{DH}$ associated with the thyrotoxicosis (1 case) and treated hyperthyroidism (3 cases). They felt that these disorders may be associated due to abnormal iodine metabolism rather than as immunologically related problems. Since then many investigators have attempted to relate $\mathrm{DH}$ to iodine metabolism, however investigation of this possible connection has led to conflicting results. Occasional reports have associated $\mathrm{DH}$ with hypothyroidism. ${ }^{1}$

$\mathrm{DH}$ has been strongly linked with HLA B8. ${ }^{10}$ However, HLA B8 has also been reported in association with gluten sensitive enteropathy, lupus erythematosus and Graves disease. ${ }^{11}$ This association could explain reports of the co-existence of $\mathrm{DH}$ and Grave's disease.

Our case also demonstrated significantly elevated thyroid antibody titers $(1: 25)$. A previous study, by Fraser found approximately $20 \%$ of patients with $\mathrm{DH}$ had thyroid microsomal antibody, compared with $2 \%$ of controls. ${ }^{12}$ This further supports a possible immunogenetic or immunological connection between these two disorders.

The association of $\mathrm{DH}$ and hyperthyroidism has been reported frequently enough to be considered more than a coincidence. Future questions need to be answered:

1. Is the identical immunoglobulin molecule involved when both conditions coexisted?

2. What is the immunofluorescent pattern in thyrotoxicosis?
3. What effect if any, does the therapy of one condition have on the course of the other?

Only when these questions are answered can we begin to understand the nature of the association of these two conditions.

\section{Drug Name}

dapsone: Avlosulfon

methimazole: Tapazole

propranolol: Inderal

\section{References}

1. Rostenberg, A., Fretzin, D., and Dimmick, D.: Dermatitis herpetiformis and hypothyroidism. Arch. Dermatol. 94:236, 1966.

2. Kumar, P. J.: Dermatitis herpetiformis associated with pernicious anemia and thyrotoxicosis. Proc. Roy. Soc. Med. 66:1128, 1973.

3. Webster, A. D. B.: Spirillum hepatitis in 'acquired' hypogammaglobulinemia with thyroiditis, pernicious anemia, and possible dermatitis herpetiformis. Proc. Roy. Soc. Med. 66:1126, 1973.

4. From, E., and Thomsen, K.: Dermatitis herpetiformis: a case provoked by iodine. Br. J. Dermatol. $91: 221,1974$

5. Douglas, W. S., and Alexander, J. O. D.: Dermatitis herpetiformis, iodine compounds and thyrotoxicosis. Br. J. Dermatol. 92:596, 1975.

6. Wise, F.: Dermatitis herpetiformis appearing three weeks after thyroidectomy. Arch. Dermatol. 27:537, 1933.

7. Goldin, D., Wilkinson, D. S.: Pretibial myxedema and thyroid acropachy. Possible dermatitis herpetiformis. Proc. Roy. Soc. Med. 66:983, 1973.

8. Rattner, H., Rodin, H., and Barsky, S.: Residual lesions of dermatitis herpetiformis and thyrotoxicosis in a woman aged 24. Arch. Dermatol. 61:704, 1950.

9. Smith, E. C.: The diagnosis of dermatitis herpetiformis. Trans. St. Johns. Dermatol. Soc. 52:176, 1966.

10. Alexander, J. O. D.: Dermatitis herpetiformis. Maj. Prob. Dermatol. 4:313, 1975.

11. Grumet, C., Konishi, J., Payne, R. O., et al.: Association of Grave's disease with HLA8. Clin. Res. 21:493, 1973.

12. Fraser, N. G.: Autoantibodies in dermatitis herpetiformis. Br. J. Dermatol. 83:609, 1970. 